\title{
A Cut-Ply Specimen for the Mixed-Mode Fracture Toughness and Fatigue Characterisation of FRPs
}

\author{
Georgia Charalambous ${ }^{*}$, Giuliano Allegri ${ }^{\xi \dagger}$, James K. Lander ${ }^{*}$, Stephen R. Hallett ${ }^{*}$ \\ *Advanced Composites Centre for Innovation and Science, University of Bristol, University Walk, \\ Bristol, BS8 1TR, UK \\ ${ }^{\dagger}$ Department of Aeronautics, Imperial College London, South Kensington Campus, \\ SW7 2AZ, London, UK \\ ${ }^{\ddagger}$ Rolls-Royce plc, The National Composites Centre, Feynman Way Central, Bristol \& Bath Science \\ Park, Emersons Green, Bristol, UK. BS16 7FS
}

\begin{abstract}
The characterization of mixed-mode fracture toughness and fatigue delamination growth in fibrereinforced composites is crucial for assessing the integrity of structural elements in service. An asymmetric cut-ply coupon (ACP) loaded in four-point bending is here proposed to carry out the aforementioned characterisations. Analytical expressions of the energy release rate and mode-mixity for the ACP are derived and validated by means of finite element analysis. A fracture toughness and fatigue characterisation of the carbon/epoxy material IM7/8552 is carried out via ACP specimens. It is proved that the material data obtained from ACP specimens match those generated using ASTM standard mixed-mode bending (MMB) coupons. The main reason for the introduction of the ACP test resides in its applicability to characterisation scenarios where measuring the delamination length with optical means, as required for MMB coupons, is difficult. Such scenarios include the investigation of static and fatigue delamination growth at low and high temperatures, which requires the usage of environmental chambers. This poses significant constraints in terms of volume available for the test rigs, and, most importantly, limitations on visual access to observe delamination propagation. However, the manufacturing of ACP coupons is more complex than for MMB specimens and the testing requires several additional precautions that are here discussed in detail.
\end{abstract}

Keywords: A. Laminates; B. Delamination; B. Fracture toughness; D. Mechanical testing

${ }^{\S}$ Corresponding Author, email: g.allegri@imperial.ac.uk, Tel. +442075945086. 


\section{Introduction}

\subsection{Literature Review}

Delamination is responsible for $60 \%$ of the structural failures of composite structural elements in service [1]. The interlaminar mixed-mode fracture toughness is usually expressed via semi-empirical laws, such as the linear interaction or the Benzeggagh-Kenane criteria [2]. Similarly, fatigue delamination growth is described by semi-empirical power laws that relate the interlaminar crack propagation rate to the energy release rate (ERR) $[3,4]$.

The ASTM standard test method for measuring the mixed-mode fracture toughness of unidirectional (UD) composites is based on the Mixed-Mode Bending (MMB) coupon [5, 6]. Alternative testing methods include the Cracked Lap Shear (CLS) [7], the Un-Symmetric Double Cantilever Beam (UDCB) [8] , the Single Leg Bend (SLB) [9], the Four-Point Bend (FPB) [10-14], the Single Leg Four Point Bend test (SLFPB) [15] and the Over-Leg Bending test (OLB)[16]. Sketches of the various testing configurations listed above are presented in Fig. 1. Each technique has advantages and drawbacks. Nonetheless, the MMB setup is still the most widely employed within the composites community due to its ASTM standardisation [6]. The MMB setup drawbacks are mainly due to the complexity and cost of the loading rig $[15,17]$. The data reduction for static MMB testing is based on beam theory and it also comprises corrections for finite laminate rotations at the delamination tip [6]. Using the MMB apparatus also involves a calibration procedure to estimate the finite compliance of the fixture. This is carried out employing a stiff prismatic and homogenous coupon and it must be repeated for several lengths of the loading arm, as described in Sec. 11.5 of the relevant ASTM standard [6]. Finally, MMB testing requires a direct optical measurement of the delamination length. CLS, SLB and OBD tests are relatively simple in terms of rig arrangements. However, the response of CLS coupons is geometrically nonlinear [15], while the mode-mixity is not constant in SLB and OBD coupons [16]. U-DCB tests also require a complex rig. The SLFPB setup is again based on four point bending, but only one delamination is allowed to propagate from a central notch via through-thickness clamping constraints [15].

FPB tests benefit from an extremely simple configuration of the loading rig and constant mode-mixity [15]. Moreover, the FPB configuration can be easily scaled. FPB tests are routinely carried out on bimaterial interfaces [10-14]. The main drawback of FPB tests is that two interlaminar cracks emanate from the central notch. These may grow at different rates, breaking the symmetry of the specimen and 
invalidating the associated analytical expression of the ERR. Lander et al. [18] manufactured CFRP FPB specimens with central surface notches by laying-up cut plies from the bottom laminate surface to the through-thickness mid-plane. They denoted such specimens as “Asymmetric Cut-Ply" (ACP) coupons, in order to differentiate these from the "central cut-ply specimens" (CCP), which had been previously employed for the characterisation of mode II fracture toughness and fatigue delamination growth in unidirectional glass/ and carbon/ epoxies [19, 20].

Regarding mixed-mode fatigue delamination growth, no standards are currently available. Several papers address the fatigue characterisation of interlaminar crack growth in FRP using MMB test setups [21-25]. This requires a preliminary static compliance calibration in order to estimate the crack length as a function of the number of load cycles during the fatigue test [24]. Alternatively the crack length can be measured "in situ" during a fatigue test using high-resolution digital images [25]. Finally, crack gauges could be bonded on the edges of the MMB coupon. The extension of other static mixed-mode characterisation techniques to fatigue has been limited so far. CLS coupons have been employed to characterise the effect of mode-mixity transition on fatigue delamination growth [26].

\subsection{Paper Overview}

This paper addresses the characterisations of the mixed-mode fracture toughness and fatigue delamination growth at room temperature for the toughened carbon/epoxy material IM7/8552. In Sec. 2, the analytical ERR and mode-mixity expressions for the ACP coupon from Ref. [18] are revisited, including the effect of the roller friction. The ACP testing setup is also extended to the characterisation of fatigue delamination growth in displacement-controlled tests and a suitable data reduction technique is introduced. In Sec. 3, experimental fracture toughness and fatigue delamination growth data obtained from ACP coupons are presented and compared to those from the MMB characterisation of the same material. In Sec. 4, the analytical ERR expressions and the large rotation correction for the ACP apparatus are validated by means of detailed finite element analyses based on a cohesive zone approach. Finally, in Sec. 5 we present a summary of the advantages and drawbacks of the ACP test setup in comparison with the MMB apparatus.

It is worth pointing out that the authors do not advocate replacing the fully standardized and wellunderstood MMB apparatus with the ACP setup. On the contrary, the goal here is to demonstrate that the ACP setup can be in principle employed to complement and extend MMB characterisations in experimental scenario where a direct measurement of the delamination length is difficult. These cases 
include low/high temperature testing where: 1) the MMB standard apparatus simply would not fit the into an environmental chamber; 2) measuring the delamination length with sufficient accuracy "in situ" is not possible due to low/high temperatures which are below/above the operational range of CCD cameras; 3 ) the usage of crack gauges is difficult due to the high cycle fatigue regime of interest, as well as the environmental conditions mentioned above. Temperature effects on fracture toughness and fatigue delamination growth are not discussed here, but they will be investigated using the ACP test setup in a future paper.

\section{The Asymmetric Cut-Ply Specimen Configuration}

The ACP coupon (Fig. 2) comprises a gauge section having thickness $t$ and length $2 L$. A delamination $2 a$ long is initiated from a surface cut, located in the middle of the gauge section. We denote with $\chi$ the ratio of the number of cut plies to the total number of layers in the UD laminate, thus $0<\chi<1$. Endtabs are adhesively bonded on the specimen, with the total thickness and length of the tabbed area being respectively $t_{T}$ and $L_{T}$. The load is applied at the end tabs via rollers, whose diameter is $D$ and whose separation distance is $d_{\mathrm{x}}$. The rollers are symmetrically placed within the end-tab areas.

\subsection{Energy release rate and mode-mixity}

Following the approach developed by Williams [27], the ACP specimen is idealised as an Euler-

Bernoulli beam. Considering the small symmetric increment of the delamination length $\Delta a$ shown in

Fig. 2, the associated change of the elastic potential energy $\Delta U$ is given by:

$$
\Delta U=\frac{M^{2}}{2 E I}\left(\frac{I}{I_{2}}-1\right) \Delta a
$$

Thus, the total ERR is given by:

$$
G=\lim _{\Delta \alpha \rightarrow 0} \frac{1}{B} \frac{\Delta U}{\Delta a}=\frac{M^{2}}{2 B E I}\left(\frac{1}{(1-\chi)^{3}}-1\right)
$$

where $E$ is the material Young's modulus in the fibre direction, $B$ is the specimen width and $M$ is the applied bending moment.

The total ERR given in Eq. (2) can be partitioned in mode I and mode II contributions. Following again Williams [27] and adopting the mode partitioning scheme illustrated in Fig. 4, the mode I and mode II bending components are given by:

$$
M_{I}=-\frac{\psi}{(1+\psi)} M \quad M_{I I}=\frac{1}{(1+\psi)} M
$$

where : 


$$
\psi=\frac{\chi^{3}}{(1-\chi)^{3}}
$$

Considering the change of the elastic potential energy associated to the modal bending components in Eq. (3), with the help of some algebra the following expressions for the mode I and mode II ERR are obtained:

$$
\begin{gathered}
G_{I}=\frac{M^{2} \psi^{2}}{2 B E I \chi^{3}(1+\psi)} \\
G_{I I}=\frac{M^{2}}{2 E I B}\left[\frac{\psi}{\chi^{3}(1+\psi)}-1\right]
\end{gathered}
$$

It is easy to check that adding up Eqs. (5) and (6) yields the total ERR in Eq. (2).

By virtue of Eqs. (2) and (6), the mode-mixity associated with the ACP coupon is expressed as

$$
\phi=\frac{G_{I I}}{G}=\frac{3(1-\chi)^{4}}{\left(1-3 \chi+3 \chi^{2}\right)\left(3-3 \chi+\chi^{2}\right)}
$$

Eq. (7) proves that the mode-mixity in ACP coupons depends only on the ratio of the number of cut plies to the total number of layers $\chi$.

\subsection{Bending moment}

The expression of the applied bending moment $M$ must include the effect of large rotations that may occur at the loading tabs. Denoting the overall load applied to the rollers as $P$, the normal force $Q$ transferred by the rollers to the end-tabs is given by (Fig. 5):

$$
Q=\frac{P}{2 \cos \beta}
$$

where $\beta$ is the rigid rotation of the end-tabs. The end-tab rotation is restricted to the

interval $\beta=\left[0 ; \frac{\pi}{2}\right]$. As shown in Fig. 5, the loading rollers and the end-tabs also exchange frictional tangential forces $\mu Q$, where $\mu$ is the Coulomb coefficient of friction. Let the following normalised constants be introduced:

$$
\alpha_{1}=\frac{D+t_{T}}{d_{x}} \quad \alpha_{2}=\frac{\mu t_{T}}{d_{x}} \quad \alpha_{3}=\frac{L_{T}}{d_{x}}
$$

It is hereby assumed that:

$$
\alpha_{1}<1
$$

Since in rolling contact usually $\mu<1$, it follows that $\alpha_{2}<\alpha_{1}$. Moreover, as shown in Fig. 2, the rollers are assumed to be located within the tabbed area, so one has $\alpha_{3}>1$. As demonstrated in Appendix A.1, the moment arm $d_{e}$ associated with the normal forces $Q$ in Fig. 5 is given by: 


$$
d_{e}=\frac{d_{x}}{\cos \beta}\left(\alpha_{1} \sin \beta-1\right)
$$

The normal forces Q produce an anti-clockwise bending moment. On the other hand, as shown in Fig. 5, the frictional forces $\mu Q$ cause a clockwise bending moment, with constant arm equal to the end-tab thickness $t_{T}$. Thus the overall bending moment acting on the specimen gauge section is expresses as follows:

$$
M=\frac{P d_{x}}{2 \cos ^{2} \beta}\left[\alpha_{1} \sin \beta-1-\alpha_{2} \cos \beta\right]
$$

Let the following normalised cross-head displacement be introduced:

$$
\bar{c}=\frac{c}{d_{x}}
$$

As demonstrated in Appendix A.1, the following relation holds between the rotation angle and the normalised cross-head displacement:

$$
\beta= \begin{cases}2 \tan ^{-1}\left(\frac{\left.1-\sqrt{1-\bar{c}\left(2 \alpha_{1}-\bar{c}\right)}\right)}{2 \alpha_{1}-\bar{c}}\right), & \bar{c}<2 \alpha_{1} \\ 2 \tan ^{-1}\left(\frac{\bar{c}}{2}\right), \quad \bar{c}=2 \alpha_{1} & \\ 2 \tan ^{-1}\left(\frac{-1+\sqrt{1+\bar{c}\left(\bar{c}-2 \alpha_{1}\right)}}{\bar{c}-2 \alpha_{1}}\right), & \bar{c}>2 \alpha_{1}\end{cases}
$$

In Appendix A.2 it is proved that under the following conditions:

$$
\alpha_{3}>1 \quad \alpha_{1}<1
$$

there exists a critical rotation angle $\left(\beta^{*}\right)$ :

$$
\beta^{*}=2 \tan ^{-1}\left(\frac{-\alpha_{1}+\sqrt{\alpha_{1}^{2}+\alpha_{3}^{2}-1}}{\left(\alpha_{3}-1\right)}\right)
$$

beyond which the rollers slip from the end-tabs, i.e. $L_{T}$ in Fig. 2 is too short for accommodating the translations of the moving roller and the tabs in the full rotation range $\beta=\left[0 ; \frac{\pi}{2}\right)$. On the other hand, for $\alpha_{3}>1$, the rollers are always in contact with the end-tabs for the aforementioned rotation range.

\subsection{Delamination length}

Considering the deformed configuration shown in Fig. 6, the local curvature of the coupon with respect to the curvilinear abscissa $s$ can be expressed as 


$$
\frac{d \theta}{d s}=\frac{12 M}{E B t^{3}(s)}
$$

where the local thickness $t(s)$ is given by:

$$
t(s)=\left\{\begin{array}{cc}
t(1-\chi) & , \quad 0 \leq s \leq a \\
t & , \quad \alpha<s \leq L
\end{array}\right.
$$

where $a$ is the delamination length. Observing that the rotation of the coupon is zero at the mid gaugelength because of symmetry and assuming that the coupon is axially inextensible, the integration of Eq. (17) yields:

$$
\theta^{*}=\frac{12 M}{E B t^{3}(1-\chi)^{3}} a \quad \beta-\theta^{*}=\frac{12 M}{E B t^{3}}(L-a)
$$

where $\theta^{*}$ is the rotation angle at the interlaminar crack tip position (see Fig. 6).

Solving Eq. (19) with respect to the crack length $a$ yields:

$$
a=\frac{1}{\bar{k}}\left(\frac{E B t^{3}}{12 M} \beta-L\right)
$$

where:

$$
\bar{k}=\frac{1-(1-\chi)^{3}}{(1-\chi)^{3}}
$$

It is worth observing that $\bar{k}>0$ in the range $0<\chi<1$.

\subsection{Stability Analysis}

For the delamination propagation to be stable in a quasi-static test the condition $d G / d a \leq 0$ must be true. By virtue of Eq. (2), the latter condition is satisfied if $d M / d a \leq 0$. Differentiating Eq. (A.5) with respect to the delamination length $a$ leads to:

$$
\frac{d \beta}{d a}=\frac{1}{\left(1+\tan ^{2} \beta\right)\left(1-\alpha_{1} \sin \beta\right)} \frac{d \bar{c}}{d a}
$$

In displacement-controlled tests, the crosshead displacement is independent from the delamination length, i.e. $d \bar{c} / d a$. Thus, Eq. (22) implies that $d \beta / d a=0$. Solving Eq. (20) with respect to the bending moment yields:

$$
M=\frac{E B t^{3}}{12(L+\bar{k} a)} \beta
$$

Differentiating eq. (23) with respect to the delamination length and considering a displacementcontrolled regime leads to: 


$$
\frac{d M}{d a}=-\bar{k} \frac{E B t^{3}}{12(L+\bar{k} a)^{2}} \beta \leq 0
$$

Since $\bar{k}>0$, Eq. (24) proves that the delamination propagation in displacement-controlled ACP coupons is unconditionally stable.

\subsection{Fatigue delamination growth}

The Fatigue Delamination Growth Rate (FDGR) can be obtained by differentiating Eq. (20), where only the maximum bending moment is a variable and all other parameters are constant. This yields:

$$
\frac{d a}{d N}=-\frac{1}{\bar{k}} \frac{E B t^{3} \beta}{12 M^{2}} \frac{d M(a)}{d N}
$$

The applied bending moment is a function of the number of cycles $N$ and it decreases as the number of cycles increases. Therefore the FDGR in ACP coupons can be computed solely from the peak force versus displacement measurements taken during a test, without the need to directly monitor the delamination length.

\section{Experimental Tests}

\subsection{Specimen preparation}

The 10 ply thick specimens used in this work were cut from a $300 \mathrm{~mm} \times 300 \mathrm{~mm}$, flat, unidirectional carbon-fibre composite plate. To produce the plate, five unidirectional plies of Hexcel IM7/8852 carbon-fibre/epoxy tape (nominal cured ply thickness of $0.125 \mathrm{~mm}$ ) were laid down in a clean environment and vacuum de-bulked for 20 minutes at room temperature to remove any trapped air. A second identical laminate was produced using the same procedure then cut in half, perpendicular to the fibre direction, using a sharp blade. These two halves were laid on top of the first laminate, tightly butted together at the cut line. An artificial pre-crack was manufactured into the laminate by including a $20 \mathrm{~mm}$ wide PTFE release film of $12.7 \mu \mathrm{m}$ thickness, laid at the mid-thickness of the laminate directly beneath the cut, as sketched in Fig. 7. The resulting laminate was subsequently de-bulked under vacuum for another 20 minutes at room temperature, prior to vacuum bagging for autoclave curing. A caul plate was used inside the vacuum bag. The resulting composite plate was then autoclaved according to the material manufacturer's recommended cure cycle.

To ensure a good grip all surfaces were grit-blasted and subsequently cleaned prior to bonding. The resulting panel was then sectioned into individual specimens, each $15 \mathrm{~mm}$ wide, using a diamond saw. Finally the cut edges of each specimen were polished using a fine grit, wet abrasive cloth. The nominal 
dimensions of the finished 5/5 surface cut ply specimens are shown in Fig. 8. The average total thickness in the tabbed region was $6.15 \mathrm{~mm}$ and the average gauge thickness was $1.25 \mathrm{~mm}$. Upon curing, the flow of resin "heals" the joining line between the cut plies. Early trials had shown that, if the resin joint was left intact, then crack initiation could not be achieved under an applied bending load. Therefore, it was necessary to fracture this resin joint so that the plies could open freely along the delaminating arms. The fracture of the resin pocket was achieved by loading the specimen in tension and immediately unloading when the first load drop was observed. Each specimen was subsequently Cscanned to check that the fracture of the resin joint did not cause any delamination propagation before the start of the tests. Two specimen configurations were tested as part of this work. In Non-PreCracked (NPC) specimens the delamination propagated directly from the edge of the release film, while the Pre-Cracked (PC) specimens emulate a naturally occurring sharp crack from which delamination growth is initiated.

Previous studies in literature have shown that, in a pure mode II regime, the interlaminar fracture toughness of IM7/8552 can be apparently enhanced when the delamination grows directly from the insert film [28]. This is the reason for testing both PC and NPC specimen configurations. The preparation of the PC specimen involved sharpening of the delamination front by propagating a short crack (nominally $5 \mathrm{~mm}$ ) beyond the edge of the release film. This was achieved by tightly clamping the specimen between four sharp edged metallic blocks, set symmetrically at $15 \mathrm{~mm}$ either side of the cut line, and then bending the specimen by hand so that a crack was driven away from the cut in both directions, up to the edge of the clamped blocks. The C-scan images of the area surrounding the cut in Fig. 9 show the limits of the release film (left) and the additional sharpened pre-crack length (right). All coupons were stored in a desiccator cabinet prior to testing to achieve a moisture-free condition.

\subsection{Test Procedure}

Following initial runs under fatigue loading, it was observed that the specimens tended to slip off the test fixture. Therefore grooved (knurled) rollers were used in order to increase the contact friction at the end-tabs, thus preventing the coupons from slipping. This is the main reason why the effect of friction was included in the bending moment calculations.

A new four-point bending test fixture was designed and manufactured to be compatible with a $1 \mathrm{kN}$ load cell. The new fixture, presented in Fig. 10, was mounted on extension rods to allow testing inside 
an environmental chamber. A video gauge was employed to check that the cross-head displacements recorded during the test were equal to the actual strokes of the loading rollers. All the static tests were conducted using an Instron 8810 series servo-hydraulic test machine at a controlled displacement rate of $1 \mathrm{~mm} / \mathrm{min}$. Time, cross-head displacement and load data were recorded continuously throughout each test. All static tests were terminated at a crosshead displacement of $10 \mathrm{~mm}$. Delamination initiation always occurred at the coupon mid-thickness plane as intended. Moreover, the two delaminations emanating from the central notch always initiated simultaneously.

Fatigue tests were run in displacement control with peak stroke corresponding to $70 \%$ of the displacement for static delamination onset, using a sinusoidal waveform with stress ratio of 0.1 and frequency of $5 \mathrm{~Hz}$. The tests were terminated after $10^{6}$ cycles.

\subsection{Quasi-static tests}

The coupons considered here consisted of 10 plies, five of which were cut. This gives $\chi=1 / 2$ and Eq. (7) yields a mode-mixity of 0.43 . Quasi-static tests were repeated on batches comprising three coupons, using NPC and PC configuration, as well as smooth and grooved (knurled) roller arrangements. Load and displacement raw data were processed using the equations presented in Sec. 2.

Fig. 11 shows the applied bending moment $(M)$ versus the cross-head displacement $(c)$ for the NPC and PC specimen configuration. Both configurations respond elastically up to the point of delamination initiation, where the maximum bending moment is reached. Following delamination onset, the crack propagated in a stable manner, marked by the plateau region in Fig. 11, until the test was terminated. The fracture toughness values $G_{C}$ obtained from the ACP coupons are presented in Tab. 1. The initiation values correspond to the peak bending moments at the beginning of the plateau regions for each test. The propagation values were computed from the average bending moments in the plateaus, up to a limit cross-head displacement of $10 \mathrm{~mm}$. Using the data presented in Ref. [29] and a linear interaction criterion for static delamination propagation would yield an initiation fracture toughness of $0.32 \mathrm{~kJ} / \mathrm{m}^{2}$ at the mode-mixity of 0.43 . Moreover, static tests performed on MMB coupons at the modemixity of 0.43 yielded an initiation fracture toughness value of $0.338 \pm 0.056 \mathrm{~kJ} / \mathrm{m}^{2}$ [4]. Using smooth rollers, the initiation $G_{C}$ values from the ACP coupons were $0.37 \pm 0.03 \mathrm{~kJ} / \mathrm{m}^{2}$ for the NPC samples and $0.35 \pm 0.01 \mathrm{~kJ} / \mathrm{m}^{2}$ for the PC coupons. Therefore the initiation fracture toughness values obtained from the ACP apparatus are in excellent agreement with those reported in the literature and based on MMB testing. 
The propagation phase showed no evident asymmetries in terms of delamination lengths with respect to the central cut. The slope of the response in the elastic region for the pre-cracked specimens is reduced because the coupons were more compliant due to the longer initial crack. Another noticeable feature associated with the delamination onset in the NPC coupons is the bending moment peak and the subsequent sudden drop at delamination initiation. The latter is an indication that a fracture toughness value taken at the delamination onset point may be influenced by the presence of the insert film. The standard deviation of the fracture toughness data in Tab. 1 for both initiation and propagation cases is within $10 \%$ of the average values. This is in line with, if not better than, what usually obtained from MMB coupons.

The introduction of grooved rollers is necessary to enable fatigue testing, since in preliminary trials it was observed that the coupons tended to slip out of the jig when cyclic loading was applied. As shown by Eq. (12), frictional forces reduce the actual bending moment on the coupon. Thus, the presence of friction may lead to an overestimation of the fracture toughness. The trouble is that it is very difficult to determine the roller friction a priori. Friction reduces the slope of the bending-moment versus displacement curve before the delamination onset. Thus, an estimation of the roller friction can be readily obtained by finding the value of $\mu$ that allows matching the slopes of the bending moment versus displacement for the smooth and grooved roller cases. For the grooved roller configuration considered here, the estimated coefficient of friction was $\mu=0.5$.

The bending moment versus crosshead displacement graph for the smooth and grooved roller configurations, including the friction correction, is presented in Fig. 12. It can be observed that the bending moment values obtained using the grooved rollers are noisier than those for the smooth rollers. Regions of fast and slow propagation can be observed, which are indicative of "stick-slip" behaviour within the roller/tab contact area. Averaging the bending moment versus the crosshead displacement still yields comparable fracture toughness values between the smooth and grooved roller cases. However, the mean fracture toughness from the tests with grooved roller is slightly larger than that obtained using smooth rollers, albeit the difference is still within one standard deviation. The effect of friction may be further reduced by optimising the roller groove pattern; a minimum $\mu$ for avoiding coupon slippage in fatigue may be determined by further experimental trials. 
In Figs. 11 and 12, no significant R-curve effect is observed. Since the average bending moment causing delamination growth remains relatively constant, it is concluded that fibre bridging is not significant while testing IM7/8552 at the mode-mixity value of 0.43 .

\subsection{Fatigue tests}

For the fatigue tests, only NPC specimens and grooved rollers were used. The data reduction technique adopted for post-processing of the fatigue data was that described in ASTM. E647-13 [30], which is based on a moving quadratic polynomial regression. This method was originally developed for metallic materials, but it has been used in the past to obtain fatigue delamination growth rates (FDGR) for FRPs [28]. The FDGR $(d a / d N)$ versus the peak ERR for UD IM7/8552 obtained from the ACP coupons at a mode-mixity of 0.43 are plotted on double logarithmic axes in Fig. 13. The peak ERR is calculated using the load value corresponding to the maximum displacement during each cycle. In Fig. 13, it can be observed that the FDGR data follow a linear trend, which can be approximated using a Paris' type power law. However, for the highest ERR values, which are closer to the material fracture toughness, one may observe the tendency of the FDGR to diverge.

Fig. 13 also presents a direct comparison of the FDGR data from the ACP tests with those obtained by IM7/8552 MMB coupons [31] at the same mode-mixity. The two testing methods yield results that are in excellent agreement. This proves that ACP specimens can provide consistent and repeatable data both for static fracture toughness and fatigue delamination growth. However, testing via the ACP coupon does not require measuring the delamination length, either directly (e.g. via digital cameras) or indirectly (via a compliance calibration).

A power-law least-square fit of the data presented in Fig. 13 yields the following semi-empirical equation:

$$
\frac{d a}{d N}=47.62\left(G_{\max }\right)^{7.4}
$$

\section{Verification using FEA}

\subsection{Finite Element Model}

For verification purposes, the analytical equations proposed in Sec. 2 are compared to the results of finite element analyses involving progressive delamination growth. A cohesive zone model was employed to describe the delamination behaviour at the continuous/cut-ply interface [32, 33]. This was done using interface elements, which follow a pre-defined traction-separation curve (Fig. 15) 
implemented in a user-defined material subroutine. The LS-Dyna explicit dynamic solver was used for the non-linear finite element analysis. In order to save computational time, only half of the ACP specimen is modelled by applying a symmetry condition at the mid-length plane (Fig. 14). A generalised plane strain state was assumed. Therefore a single element mesh was employed in the width direction.

The nominal specimen geometry was considered in the FE simulations, with cohesive elements of $0.01 \mathrm{~mm}$ thickness at the delaminating interface. The contact between the rollers and the end-tabs was represented in detail in the FE models.

Three-dimensional, eight-node solid elements with full integration were used for the composite plies, while constant stress interface elements were employed to model the cohesive zone. The element length was kept at $0.03 \mathrm{~mm}$ for a distance of $15 \mathrm{~mm}$ from the central cut, after which the element size was increased using a biased mesh to a maximum length of $0.5 \mathrm{~mm}$ at the end of the gauge section. The composite plies were assumed linear elastic and orthotropic with properties summarised in Tab. 2 [3437]. The cohesive element properties are listed in Tab. 3 [32-33]. The bilinear traction separation curve simulating the constitutive behaviour of the user-defined cohesive elements is defined according to a quadratic stress-based initiation criterion and a linear ERR-based damage propagation criterion [32, 33]. The ERR at each time-step (mode I, mode II and total) is calculated from the integration of the corresponding cohesive stresses versus interface separations.

\subsection{Simulation Results}

In order for the simulation results to be directly comparable to the analytical ERR formulae, a fully developed "process zone" must be formed ahead of the interlaminar crack tip [32]. This should comprise several elements undergoing progressive damage accumulation. The formation of a process zone represents delamination initiation. Once the "process zone" is fully developed, it will translate in a self-similar fashion as the delamination propagates. For the current model, the fully developed process zone was found to be $0.25 \mathrm{~mm}$ long.

Fig. 16 presents the ERRs and associated mode mixity at the crack tip versus the delamination length. The values are computed at the first fully failed element behind the process zone. It can be observed that constant mode-mixity and critical ERR (i.e. propagation) values are obtained after delamination initiation, i.e. for delamination lengths larger than $0.25 \mathrm{~mm}$. Therefore the analytical results yielding a 
constant mode-mixity in the gauge section were verified by means of cohesive zone modelling, as summarised in Tab. 4. The difference in the fracture toughness value yielded by the FE analysis is due to the fact that, as mentioned before, the cohesive zone model is based on a linear ERR interaction criterion for predicting damage propagation. Thus, the mixed-mode fracture toughness in the simulations is calculated using the mode I and mode II values given in Tab. 3. As mentioned before, for a combination of $G_{\mathrm{IC}} / G_{I \mathrm{C}}$ of $0.24 / 0.78(\mathrm{~kJ} / \mathrm{m} 2)$, the predicted fracture toughness according to a linear interaction criterion is $0.34 \mathrm{~N} / \mathrm{mm}$, which is $10.5 \%$ lower than that found experimentally. Other sources in the literature indicate that $G_{\mathrm{IIC}}$ for IM7/8552 can be as high as $1.2 \mathrm{~kJ} / \mathrm{m}^{2}$ [29]; in this case the predicted fracture toughness would be $0.37 \mathrm{~N} / \mathrm{mm}$ which is closer to the measured experimental value $(0.38 \mathrm{~N} / \mathrm{mm})$. It was observed that the first failure in the finite element models occurred at a bending moment per unit width of $49 \mathrm{~N}$ (Tab. 4). In the experiments, the average bending moment per unit width at initiation was $50.86 \mathrm{~N}$. Therefore the difference between the two values is negligible. In order to verify the analytical prediction of the effective bending moment arm $\left(d_{e}\right)$, a master-slave contact model was used to simulate the interaction between the rollers and the tabs. For a crosshead displacement of $7 \mathrm{~mm}$, the FE model yielded a measured bending moment arm of $21.01 \mathrm{~mm}$, which is in excellent correlation with the $21.09 \mathrm{~mm}$ predicted from the analytical solution. Hence, the large rotation correction suggested for calculation of the effective bending moment arm is well suited for describing the actual coupon kinematics.

\section{Discussion}

The analyses carried out in Secs. 2 and 4, as well as the experimental results discussed in Sec. 3, demonstrate that the ACP test setup is characterised by ERR values independent from the delamination size along the whole gauge length. In a displacement-controlled regime, the delamination growth is also intrinsically stable. Moreover, the actual delamination length can be easily calculated from the machine crosshead displacement. This represents an advantage, particularly in terms of fatigue testing. Nonetheless, the preparation of ACP coupons is more complex than that of MMB specimens. Cutting the plies constitutes an additional manufacturing step. Fracturing the resin bridge at the cut location after cure requires loading the specimen in tension in a servo-hydraulic machine prior to the actual testing. This may indeed cause some delamination propagation; thus, C-scans are required to verify that the interlaminar crack tips are still located at the ends of the release film after the resin bridge has been 
fractured. These additional complications can be avoided by creating a gap a few millimetres wide between the cut plies, in order to prevent the formation of a continuous resin bridge. The presence of such a gap will not influence the fracture behaviour of the ACP coupon and it will considerably ease the specimen preparation. However, tabs will still be needed in order to ensure that the bending and shear deformations of the coupons between the loading and support rollers will be negligible, otherwise the large rotation correction introduced in Sec 2.2 to calculate the actual bending moment would be invalid.

The mode-mixity ratio can be adjusted by varying the number of cut plies for a given specimen thickness. This implies that a different panel will have to be manufactured for each mode-mixity value considered, potentially leading to increased costs.

Regarding the actual testing procedure, it is mandatory to achieve a symmetrical delamination growth in the gauge section. This requires reasonably good dimensional tolerances for the finished coupons as well as a careful alignment of the samples in the four-point bending rig. The coupons described in Sec 3 were all well within 1 decimal place dimensional standard tolerance (DST) in terms of length and width and 2 decimal places DST for the thickness. The correct alignment of the coupon in the rig was achieved using a custom-made graduated bar.

Overall, the ACP configuration may represent a viable alternative to the usage of an MMB apparatus in cases where measuring the delamination length is particularly difficult and/or the space available is limited. These include testing in environmental chambers at temperatures, both low and high, that exceed the operational range of cameras and video gauges.

\section{Conclusions}

Mixed-mode static and fatigue tests were carried out on the IM7/8552 carbon-epoxy composite using asymmetric cut-ply (ACP) coupons loaded in four-point bending. It has been proven that consistent and repeatable values of the interlaminar fracture toughness and FDGR data can be obtained without measuring directly the interlaminar crack length or adopting a compliance calibration procedure.

Therefore, the ACP setup may represent a suitable alternative to the well-understood and standardised MMB apparatus for testing scenarios where a visual measurement of the delamination length proves particularly difficult, as in the characterisation of temperature effects on static and fatigue delamination growth, which has to be carried out within environmental chambers. 
A wide range of mode-mixities can be characterised solely by changing the ratio of the number of cut plies to the total number of plies. However, this requires manufacturing a different unidirectional plate for each mode-mixity. As the ERR values are proportional to the square of the applied bending moment, the latter must be corrected for the large rotations occurring at the end-tabs. The presence of grooved (knurled) rollers and the related effect has been also included in the analytical ERR expression and a simple method for estimating the associated friction coefficient has been described and validated. Finite element simulations of the ACP models showed that the mode mixity is kept constant across the process zone and the associated value matches the prediction from the analytical solution. Moreover, the FE analyses prove the accuracy of the large rotation correction introduced for estimating the actual bending moment applied on the specimen.

However, the manufacturing of ACP coupons requires special care to ensure that strict dimensional tolerances are met. Fracturing the resin bridge between the cut plies requires special attention in order to avoid the initiation of delaminations. Achieving symmetric delamination growth in the gauge section needs a very careful alignment of the coupon in the four-point bending rig.

Two ACP specimen configurations have been investigated in this study, i.e. considering the delamination growth directly from the insert film (NPC specimens) and from a naturally occurring sharp crack (PC specimens). The difference between the average initiation and propagation fracture toughness for the two cases is statistically insignificant. Under cyclic loading, the fatigue delamination growth rate data obtained using the ACP testing matched those generated using MMB coupons.

\section{Acknowledgements}

The authors would like to acknowledge Rolls-Royce plc for the support to this research granted via the Composites University Technology Centre of the University of Bristol, UK.

\section{References}

[1] A.G. Miller, D.T. Lovell, J.C. Seferis, The evolution of an aerospace material: Influence of design, manufacturing and in-service performance, Composite Structures, 27 (1994) 193-206.

[2] M.L. Benzeggagh, M. Kenane, Measurement of mixed-mode delamination fracture toughness of unidirectional glass/epoxy composites with mixed-mode bending apparatus, Composites Science and Technology, 56 (1996) 439-449.

[3] T.K. O'Brien, Toward a damage tolerance philosophy for composite materials and structures, ASTM, Composite Materials: Testing and Design. Ninth Volume, 1990 (1990) 7-33.

[4] G. Allegri, M.R. Wisnom, S.R. Hallett, A new semi-empirical law for variable stress-ratio and mixed-mode fatigue delamination growth, Composites Part A: Applied Science and Manufacturing, 48 (2013) 192-200. 
[5] J.R. Reeder, J.H. Rews, Mixed-mode bending method for delamination testing, AAAA Journal, 28 (1990) 1270-1276.

[6] ASTM D6671 / D6671M-06 -Standard Test Method for Mixed Mode I-Mode II Interlaminar Fracture Toughness of Unidirectional Fiber Reinforced Polymer Matrix Composites, in, ASTM International.

[7] T.R. Brussat, S.T. Chiu, S. Mostovoy, Fracture mechanics for structural adhesive bonds, Air Force Material Laboratory, Technical Report 77-163, 1977.

[8] V. Sundararaman, B.D. Davidson, An unsymmetric double cantilever beam test for interfacial fracture toughness determination, International journal of solids and structures, 34 (1997) 799-817. [9] B.D. Davidson, V. Sundararaman, A single leg bending test for interfacial fracture toughness determination, International Journal of Fracture, 78 (1996) 193-210.

[10] H.C. Cao, A.G. Evans, An experimental study of the fracture resistance of bimaterial interfaces, Mechanics of Materials, 7 (1989) 295-304.

[11] P.P.L. Matos, R.M. McMeeking, P.G. Charalambides, M.D. Drory, A method for calculating stress intensities in bimaterial fracture, International Journal of Fracture, 40 (1989) 235-254.

[12] Z. Suo, Delamination specimens for orthotropic materials, ASME Transactions - Journal of Applied Mechanics, 57 (1990) 627-634.

[13] A.G. Evans, M. Rühle, B.J. Dalgleish, P.G. Charalambides, The fracture energy of bimaterial interfaces, Materials Science and Engineering: A, 126 (1990) 53-64.

[14] I. Hofinger, M. Oechsner, H.-A. Bahr, M.V. Swain, Modified four-point bending specimen for determining the interface fracture energy for thin, brittle layers, International Journal of Fracture, 92 (1998) 213-220.

[15] G.D. Tracy, P. Feraboli, K.T. Kedward, A new mixed mode test for carbon/epoxy composite systems, Composites Part A: Applied Science and Manufacturing, 34 (2003) 1125-1131.

[16] A. Szekrényes, U.J. József, Over-leg bending test for mixed-mode I/II interlaminar fracture in composite laminates, International Journal of Damage Mechanics, 16 (2007) 5-33.

[17] A.J. Brunner, B.R.K. Blackman, P. Davies, A status report on delamination resistance testing of polymer-matrix composites, Engineering Fracture Mechanics, 75 (2008) 2779-2794.

[18] J.K. Lander, L.F. Kawashita, G. Allegri, S.R. Hallet, M.R. Wisnom, A cut ply specimen for the determination of mixed-mode interlaminar fracture toughness, in: 14th European Conference on Composite Materials, Budapest, Hungary, 2010.

[19] M.R. Wisnom, M.I. Jones, W. Cui, Delamination in composites with terminating internal plies under tension fatigue loading, ASTM STP 1230, (1995) 486-508.

[20] G. Allegri, M.I. Jones, M.R. Wisnom, S.R. Hallett, A new semi-empirical model for stress ratio effect on mode II fatigue delamination growth, Composites Part A: Applied Science and Manufacturing, 42 (2011) 733-740.

[21] S. Zhao, M. Gadke, R. Prinz, Mixed-mode delamination behavior of carbon/epoxy composites, Journal of reinforced plastics and composites, 14 (1995) 804-826.

[22] L.E. Asp, A. Sjögren, E.S. Greenhalgh, Delamination growth and thresholds in a carbon/epoxy composite under fatigue loading, Journal of composites technology \& research, 23 (2001) 55-68.

[23] N. Blanco, E.K. Gamstedt, L.E. Asp, J. Costa, Mixed-mode delamination growth in carbon-fibre composite laminates under cyclic loading, International Journal of Solids and Structures, 41 (2004) 4219-4235.

[24] M. Kenane, M.L. Benzeggagh, Mixed-mode delamination fracture toughness of unidirectional glass/epoxy composites under fatigue loading, Composites Science and Technology, 57 (1997) 597605.

[25] D. Tumino, B. Zuccarello, Fatigue delamination experiments on GFRP and CFRP specimens under single and mixed fracture modes, Procedia Engineering, 10 (2011) 1791-96.

[26] R.L. Ramkumar, J.D. Whitcomb, Characterization of mode I and mixed-mode delamination growth in T300/5208 graphite/epoxy. In: Delamination and debonding of materials, ASTM STP 876, (1985) 315-335.

[27] J.G. Williams, On the calculation of energy release rates for cracked laminates, International Journal of Fracture, 36 (1988) 101-119.

[28] T.K. O'Brien, W.M. Johnston, G.J. Toland, Mode II interlaminar fracture toughness and fatigue characterization of a graphite epoxy composite material, NASA/TM 216838, 2010.

[29] P. Hansen, R. Martin, DCB, 4ENF and MMB delamination characterisation of S2/8552 and IM7/8552, Technical Report N68171-98-M-5177, Materials Engineering Research Laboratory Ltd. (MERL), Hertford, UK ,1999..

[30] ASTM E647, Standard Test Method for Measurement of Fatigue Crack Growth Rate, In: ASTM International, 2013. 
[31] S. Giannis, Utilising fracture mechanics principles for predicting the mixed-mode delamination onset and growth in tapered composite laminates, Composite Structures, 102 (2013) 294-305.

[32] W.G. Jiang, S.R. Hallett, B.G. Green, M.R. Wisnom, A concise interface constitutive law for analysis of delamination and splitting in composite materials and its application to scaled notched tensile specimens, International Journal for Numerical Methods in Engineering, 69 (2007) 1982-1995.

[33] P.W. Harper, S.R. Hallett, A fatigue degradation law for cohesive interface elements-

Development and application to composite materials, International Journal of Fatigue, 32 (2010) 1774 1787.

[34] T.K. O'Brien, R. Krueger, Analysis of ninety degree flexure tests for characterization of composite transverse tensile strength, NASA/TM 211227, ARL-TR-2568, (2001).

[35] G.B. Murri, Evaluation of Delamination Growth Characterization Methods Under Mode I Fatigue Loading, ASC $27^{\text {th }}$ Technical Conference, ASTM D30 Meeting, Arlington, TX (2012).

[36] Hexcel Composites, Product Data HexPly 8552 Epoxy matrix, available online at URL http://www.hexcel.com/Resources/DataSheets/Prepreg-Data-Sheets/8552 eu.pdf (last retrieved November 2014).

[37] J. Lee, C. Soutis, Measuring the notched compressive strength of composite laminates: Specimen size effects, Composites Science and Technology, 68 (2008) 2359-2366.

\section{Appendices}

\section{A.1 Large rotation kinematics}

The coordinates of the roller/tab contact points in Fig. 5 with respect to the centre of the support (lower) roller are

$$
\begin{aligned}
& C_{1} \equiv\left[-d_{x}+\frac{D}{2} \sin \beta ; t_{T}+D\left(1-\frac{1}{2} \cos \beta\right)\right] \\
& C_{2} \equiv\left[-\frac{D}{2} \sin \beta ; c+\frac{D}{2} \cos \beta\right]
\end{aligned}
$$

Consequently, the vector connecting the contact points is:

$$
\underline{C_{1} C_{2}}=\left[d_{x}-D \sin \beta ; c-t_{T}-D(1-\cos \beta)\right]
$$

Similarly, in Fig. 5, the coordinate of the points lying on the contact normal at the mid throughthickness plane of the end-tabs are:

and the associated vector is:

$$
\begin{aligned}
& S_{1} \equiv\left[-d_{x}+\frac{1}{2}\left(D+t_{T}\right) \sin \beta ;\left(D+t_{T}\right)\left(1-\frac{1}{2} \cos \beta\right)\right] \\
& S_{2} \equiv\left[-\frac{1}{2}\left(D+t_{T}\right) \sin \beta ; c+\frac{1}{2}\left(D+t_{T}\right) \cos \beta\right]
\end{aligned}
$$

$$
\underline{S_{1} S_{2}}=\left[d_{x}-\left(D+t_{T}\right) \sin \beta ; c-\left(D+t_{T}\right)(1-\cos \beta)\right]
$$

From Fig. 5, one can observe that the slope of the segment $S_{1} S_{2}$ is $\tan \beta$. Thus, from Eq. (A.4) and the definition of the normalised constants in Eqs. (9) and (13), one can write:

$$
\tan \beta=\frac{\bar{c}-\alpha_{1}(1-\cos \beta)}{1-\alpha_{1} \sin \beta}
$$

Eq. (A.5) expresses the end-tab rotation as a function of the normalised crosshead displacement $\bar{c}$.

Having assumed $\alpha_{1}<1$ implies that the denominator on the right hand side of Eq. (A.5) is always 
positive for $\beta \in\left[0 ; \frac{\pi}{2}\right)$. The distance $d_{e}$ that represents the moment arm of the normal forces exerted by the rollers on the end-tabs can be found as follows:

$$
d_{e}=\underline{C_{1} C_{2}} \cdot \underline{k^{\prime}}
$$

where $k^{\prime}$ is the versor of the specimen axis, whose components are:

$$
\underline{k^{\prime}}=[\cos \beta ; \sin \beta]
$$

Substituting Eqs. (A.2) and (A.7) into Eq. (A.6) yields:

$$
d_{e}=d_{x}\left(\cos \beta+\bar{c} \sin \beta-\alpha_{1} \sin \beta\right)
$$

Solving Eq. (A.5) with respect to $\bar{c}$ and substituting the result into Eq. (A.8), with the help of some algebra, leads to Eq. (11).

\section{A.2 Limit rotation angle}

For a given specimen/rig configuration, a limit rotation angle $\beta^{*}<\frac{\pi}{2}$ may be reached when the rollers are both located on the end-tab edges, i.e. $d_{e}=L_{T}$. Any further increment of the rotation angle beyond $\beta^{*}$ would cause the rollers to slip from the end tabs. The limit condition $d_{e}=L_{T}$ can be expressed by means of Eq. (A.8) as:

$$
\alpha_{1} \sin \beta^{*}-\alpha_{3} \cos \beta^{*}=1
$$

where the definitions of normalised parameters given in Eq. (9) have been employed.

Employing the Weierstrass substitution

$$
u^{*}=\tan \frac{\beta^{*}}{2}
$$

allows solving Eq. (A.9) with respect to $u^{*}$ and it leads to

$$
u^{*}=\frac{-\alpha_{1}+\sqrt{\alpha_{1}^{2}+\alpha_{3}^{2}-1}}{\left(\alpha_{3}-1\right)}
$$

Eq. (A.10) yields Eq. (16). Since $\alpha_{1}<1$ and $\alpha_{3}>1$, from Eq. (A.11) a limit rotation angle $\beta^{*}<\frac{\pi}{2}$ exists only if 


$$
\left(\frac{-\alpha_{1}+\sqrt{\alpha_{1}^{2}+\alpha_{3}^{2}-1}}{\left(\alpha_{3}-1\right)}\right)<1
$$

which, with the help of some algebra, leads to the conditions stated in Eq. (15). On the other hand, if the inequalities in Eq. (15) are not met, the rollers cannot slip from the end-tabs for $\beta \in\left[0 ; \frac{\pi}{2}\right)$.

\section{A.3 Rotation angle as a function of the crosshead displacement}

Using again the Weierstrass substitution, Eq. (A.5) can be solved in order to obtain an explicit relation between the rotation angle $\beta$ and the normalised crosshead displacement $\bar{c}$. This leads to the following quartic equation:

$$
\left(u^{2}+1\right)\left[\left(2 \alpha_{1}-\bar{c}\right) u^{2}-2 u+\bar{c}\right]=0
$$

The first quadratic factor in Eq. (A.13) has only imaginary roots. The discriminant associated with the second quadratic factor is:

$$
\Delta=1-2 \alpha_{1}(\bar{c})+(\bar{c})^{2}
$$

Setting the discriminant in Eq. (A.14) to zero yields the normalised crosshead displacements

$$
\bar{c}_{1,2}=\alpha_{1} \pm \sqrt{\alpha_{1}^{2}-1}
$$

which are complex valued. Since the sign of the leading term in the right hand side of Eq. (A.14) is positive, it follows that the determinant (A.14) is always positive. Thus Eq. (A.13) admits only two real roots for $\alpha_{1}<1$. If $\bar{c}<2 \alpha_{1}$, the roots are both positive. However, the larger root corresponds to a

rotation angle $\beta>\frac{\pi}{2}$ and therefore it can be discounted. Thus, for $\bar{c}<2 \alpha_{1}$, the rotation angle is given by the first of Eqs. (14) In the case $\bar{c}=2 \alpha_{1}$, the real roots are coincident and given by the second of Eqs. (14). Finally, for $\bar{c}>2 \alpha_{1}$, only one positive root exists. The positive root in this last case is given by the third of Eqs. (14). 


\section{Figures}
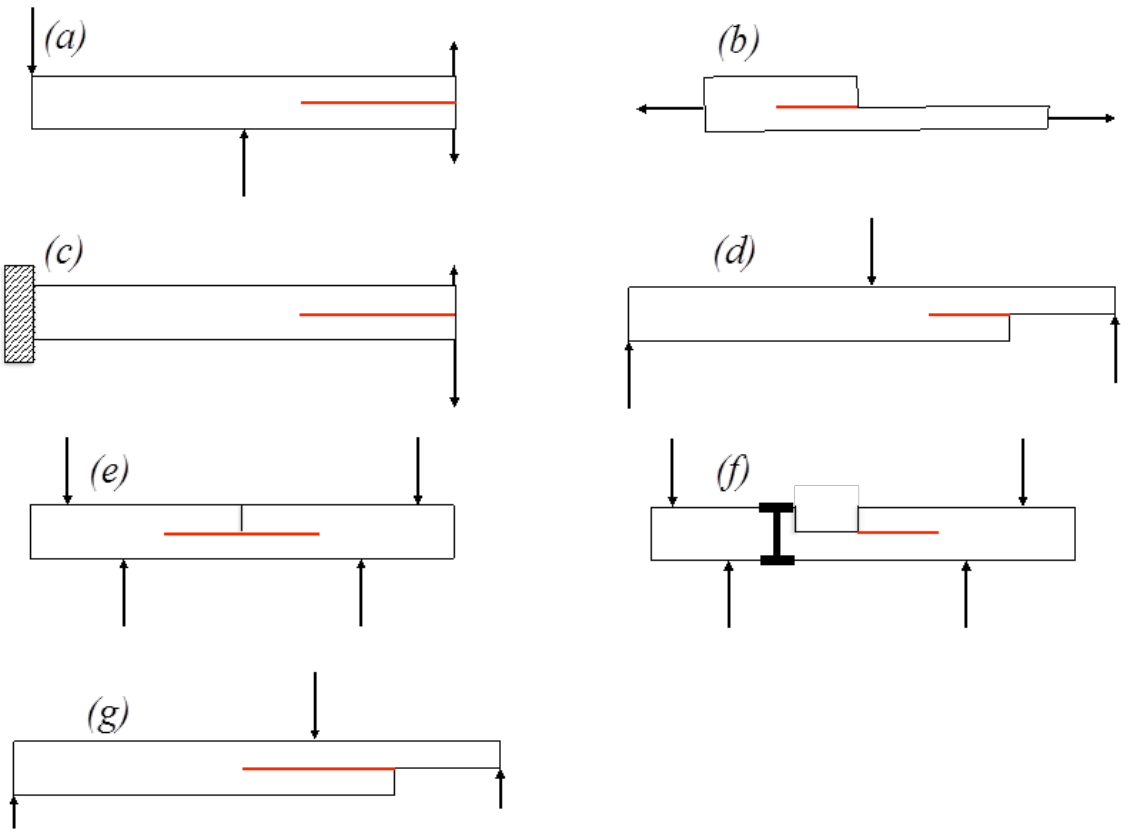

Figure 1: Specimen configurations for mixed-mode static/fatigue testing: a) mixed-mode bending (MMB)[5, 6]; b) cracked lap shear (CLS)[7]; c) un-symmetric double cantilever beam (U-DCB)[8]; d)

single leg bend (SLB)[9]; e) four point bending (FPB)[10-14]; (f) single leg four point bending

(SLFPB)[15]; (g) over-leg bending (OLB)[16].

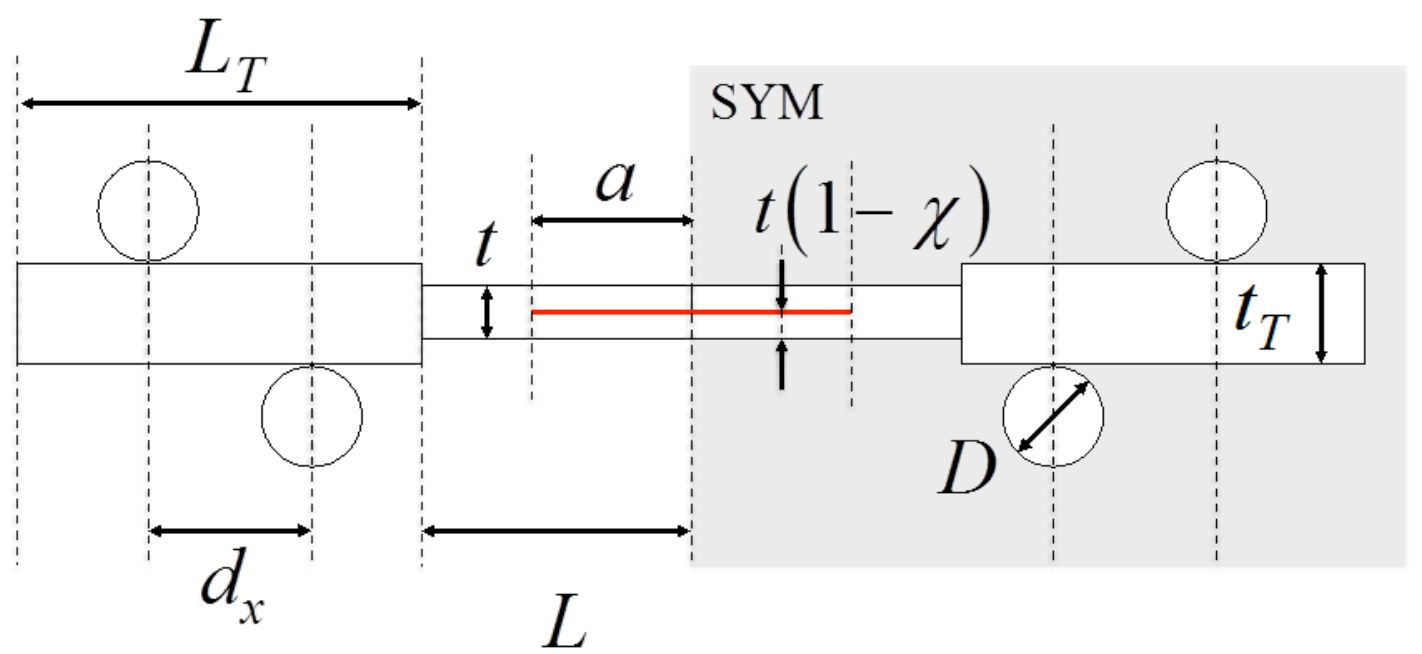

Figure 2: Configuration of the asymmetric cut-ply specimen (ACP) 


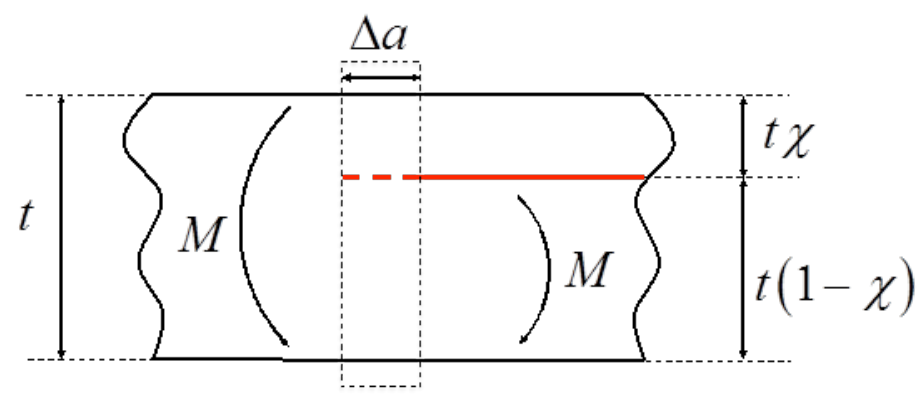

Figure 3: Delamination extension for ERR calculations.

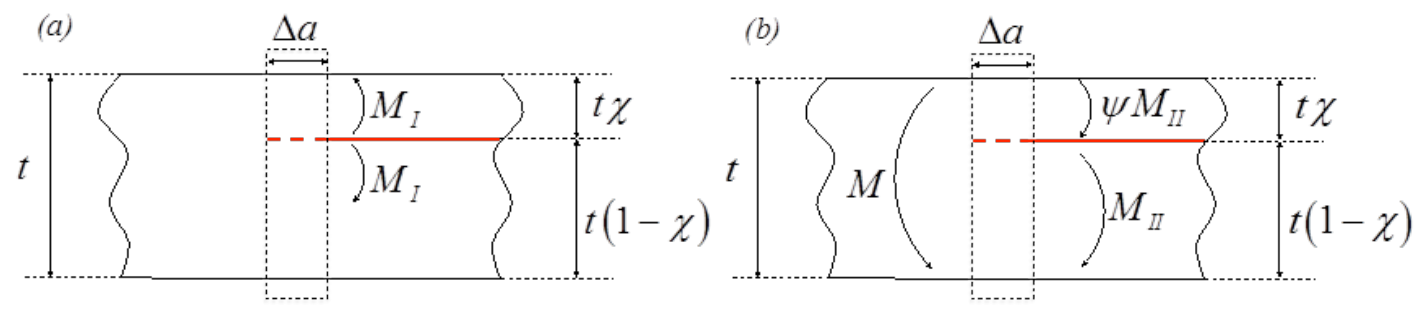

Figure 4: Partition of delamination modes; (a) mode I; (b) mode II. 


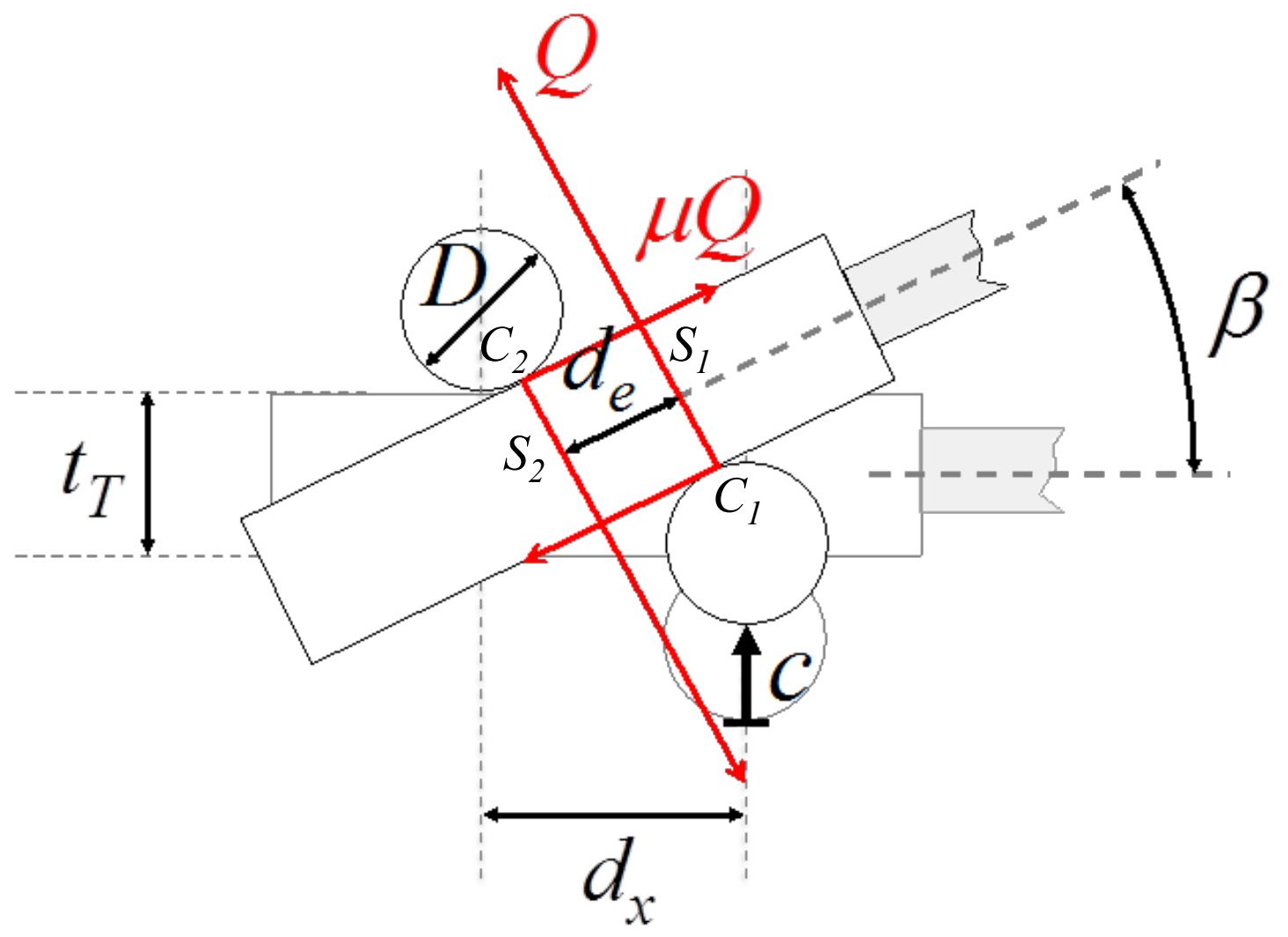

Figure 5: Contact forces and moment arms for large end tab rotations.

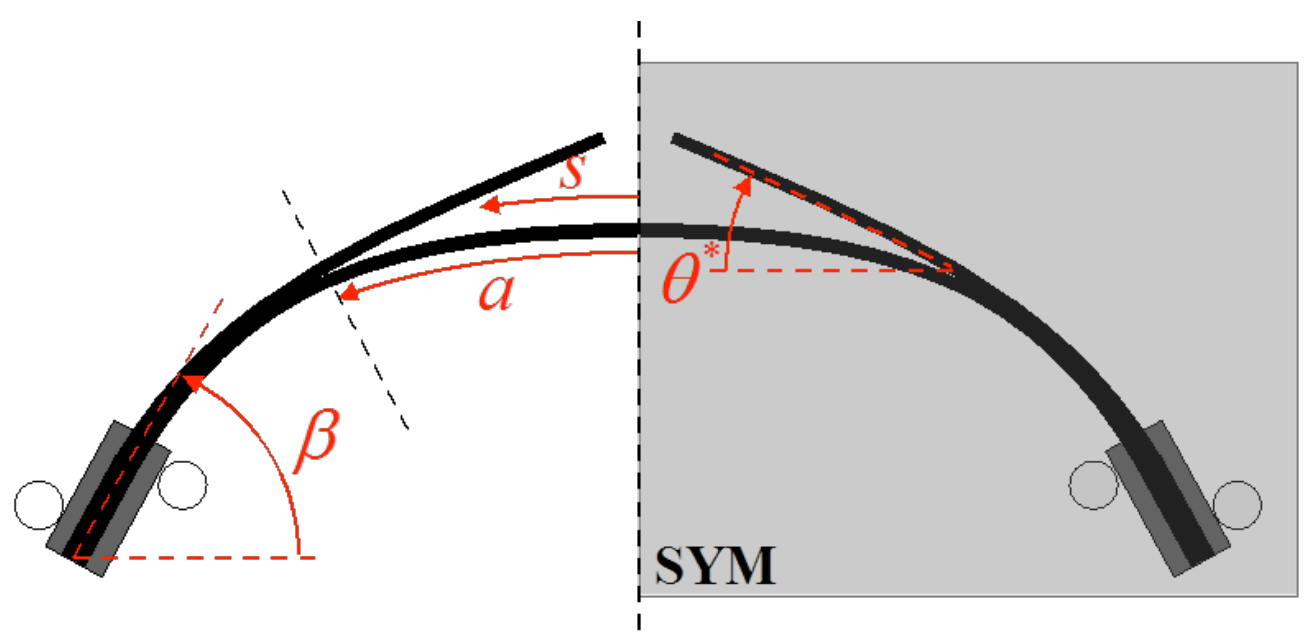

Figure 6: Deformed configuration. 


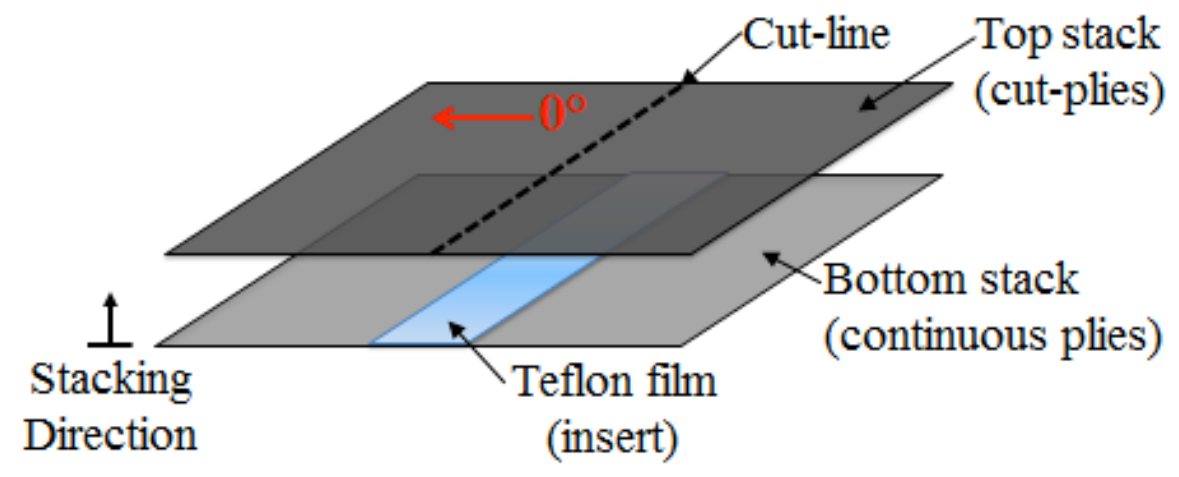

Figure 7: Exploded surface cut-ply laminate lay-up.

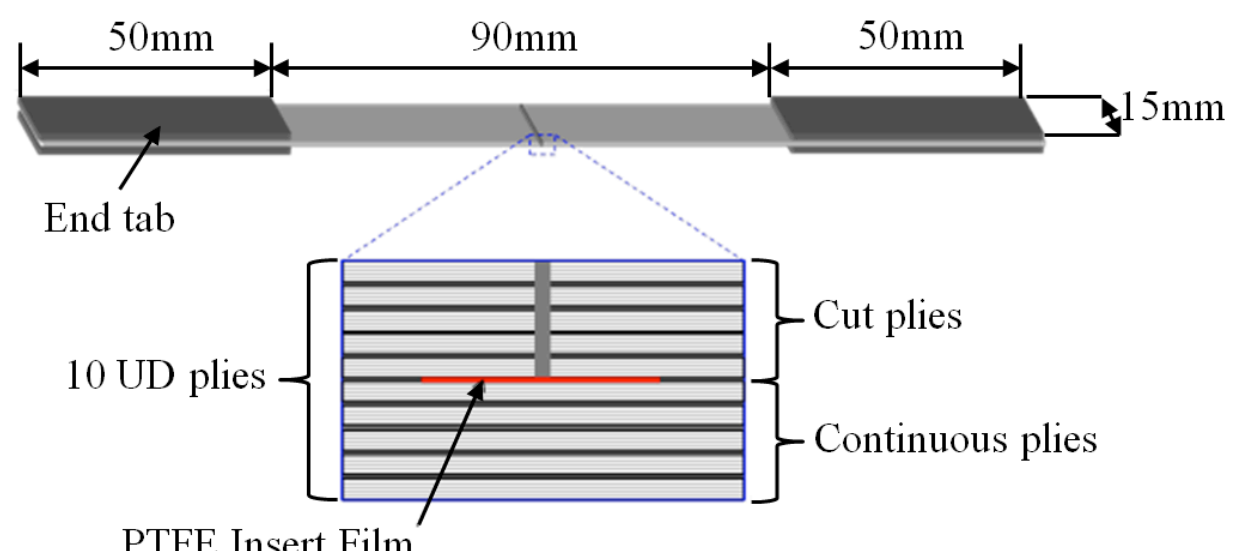

Figure 8: Schematic diagram of the University of Bristol Asymmetric Cut Ply (ACP) specimen. 

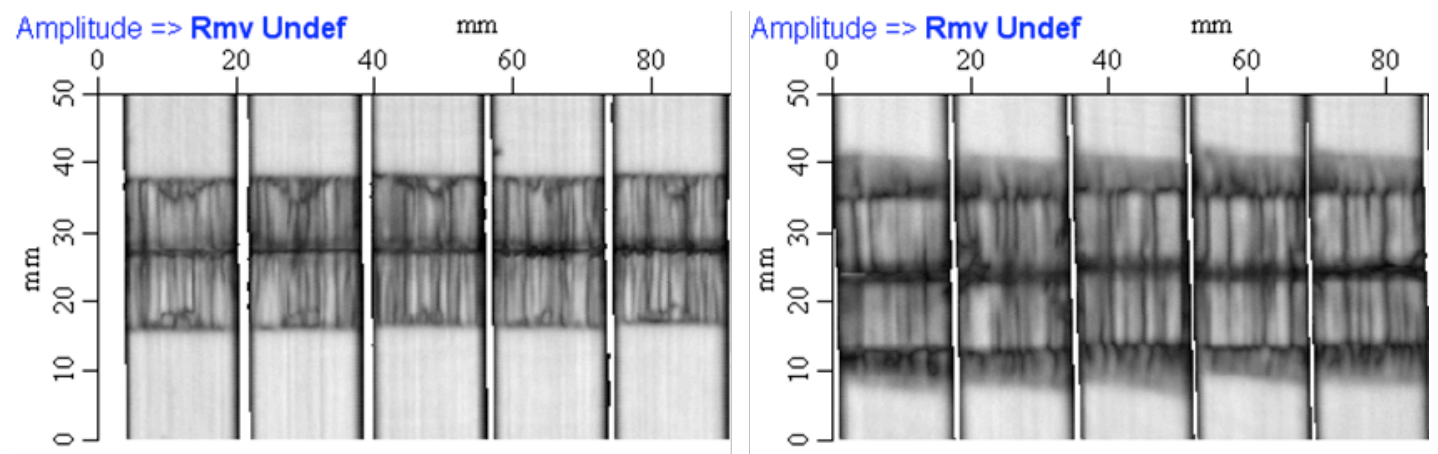

Figure 9: C-scans of the cut ply region showing the release film boundaries for the Non Pre-Cracked (NPC) specimens (left) and the additional pre-crack distance in the Pre-Cracked (PC) specimens (right).

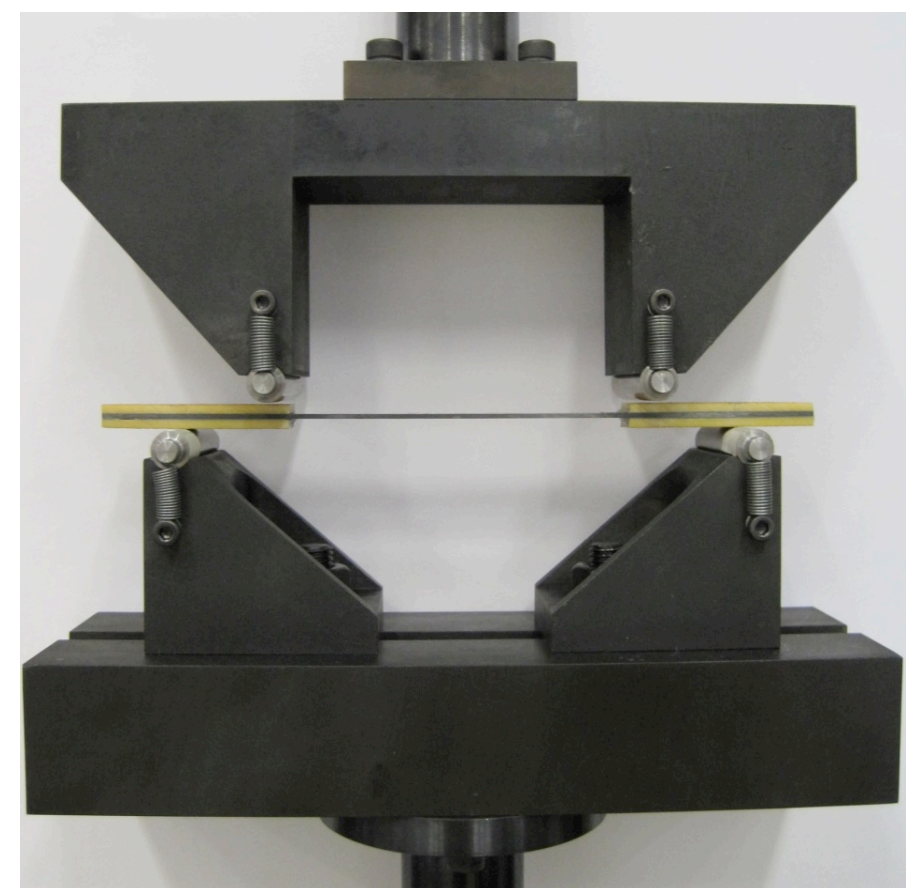

Figure 10: Four-point bending fixture used for testing ACP coupons. 


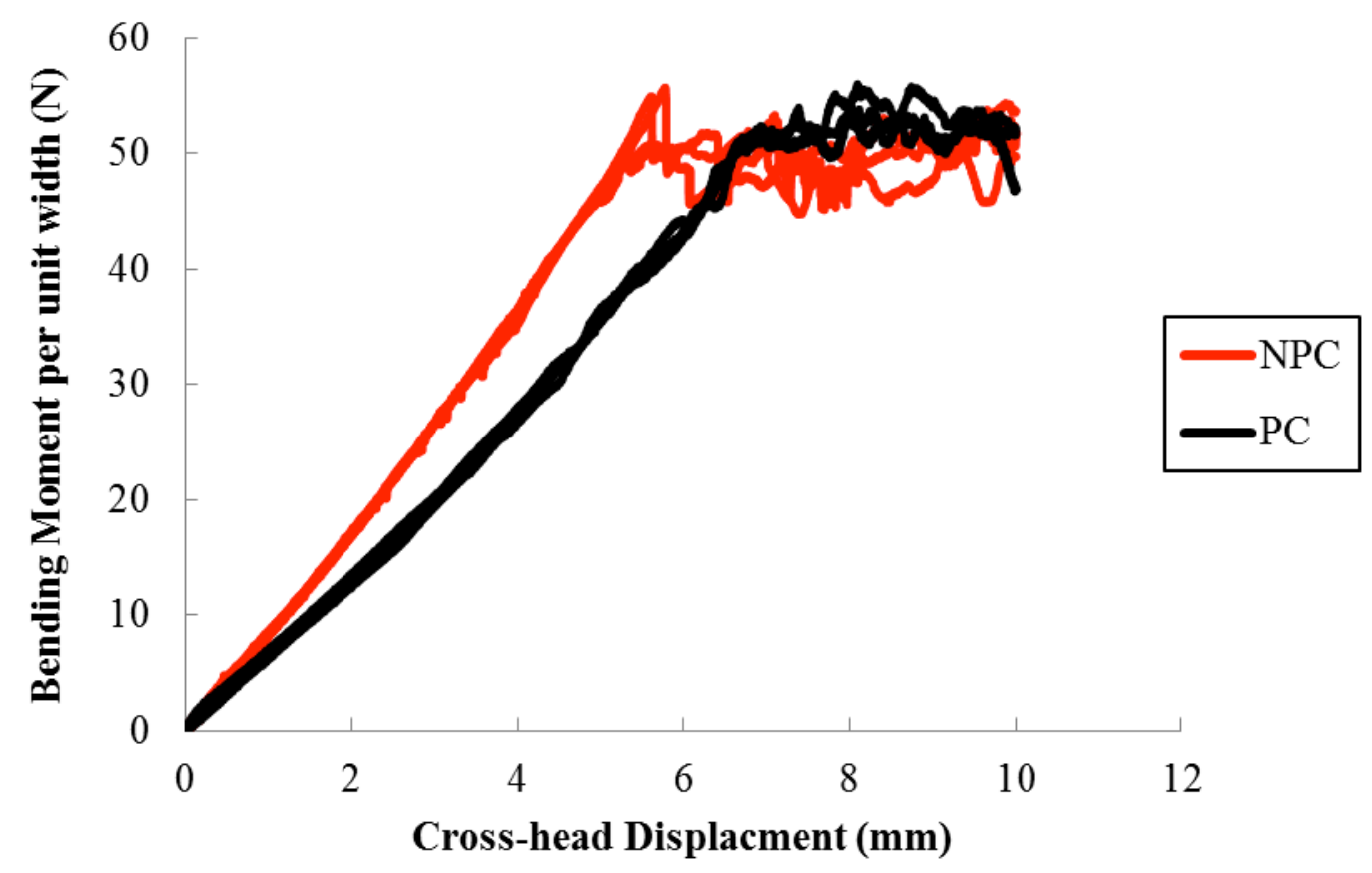

Figure 11: Bending Moment per unit width $(M / B)$ vs crosshead displacement $(c)$ obtained using smooth rollers.

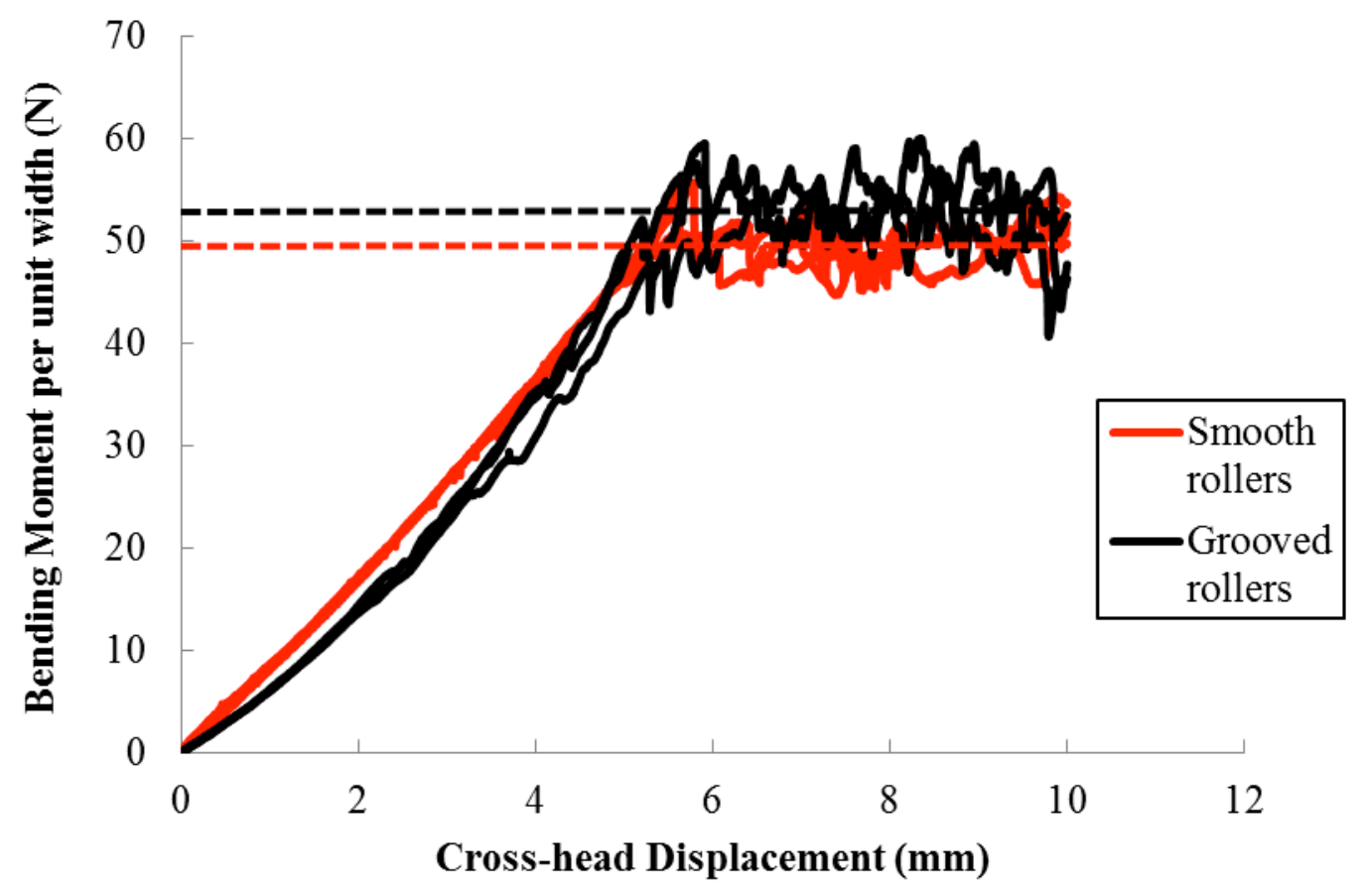

Figure 12: Comparison of bending moment per unit width $(M / B)$ vs crosshead displacement $(c)$ obtained using smooth and grooved rollers (after friction correction). 


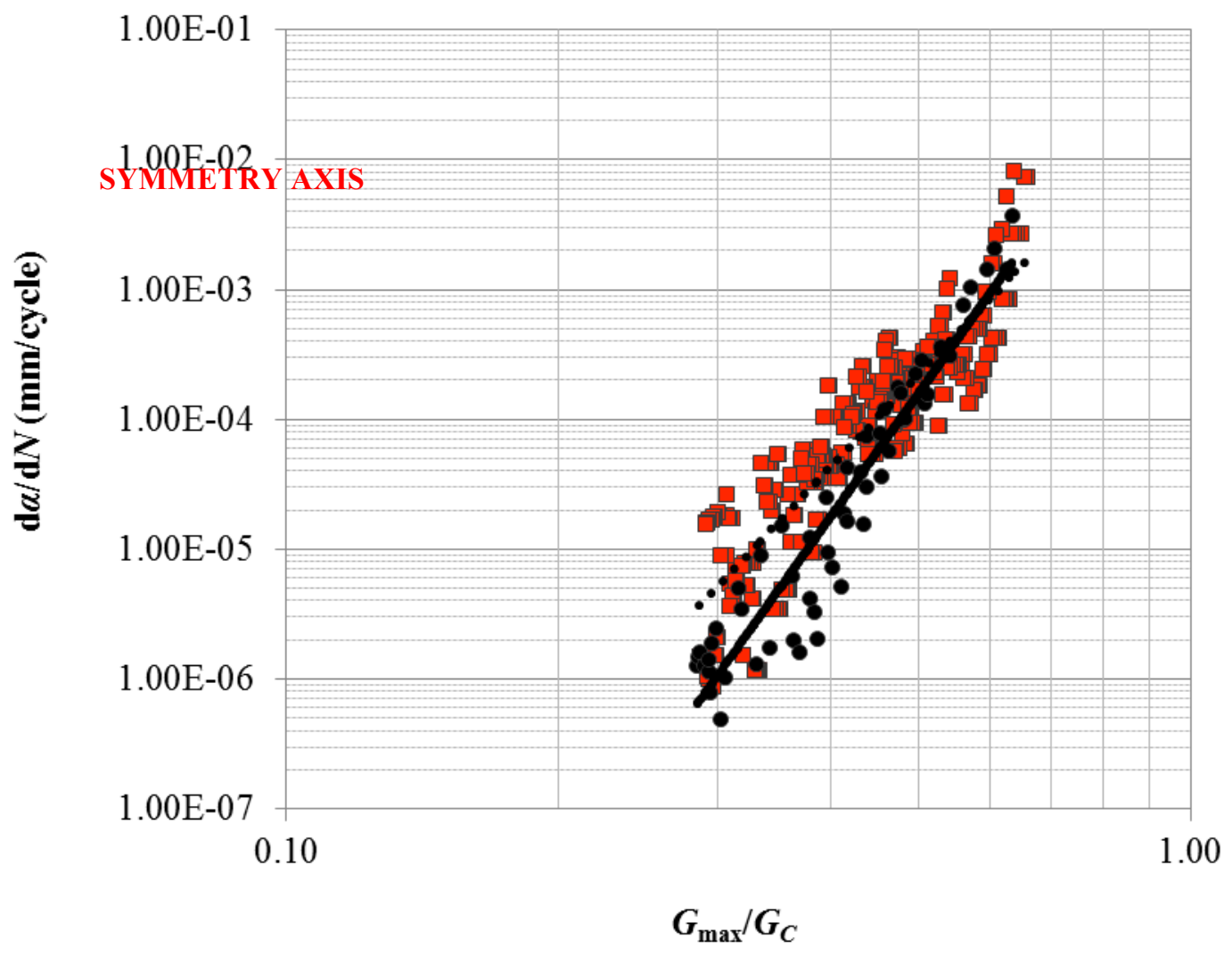

- $\mathrm{ACP} \quad$ - $\mathrm{MMB} \quad \cdots$. Power (ACP) $\longrightarrow$ Power (MMB)

Figure 13: Fatigue delamination growth rates versus peak ERR. The red squares represent the ACP data, while the black circles are from MMB tests [4].

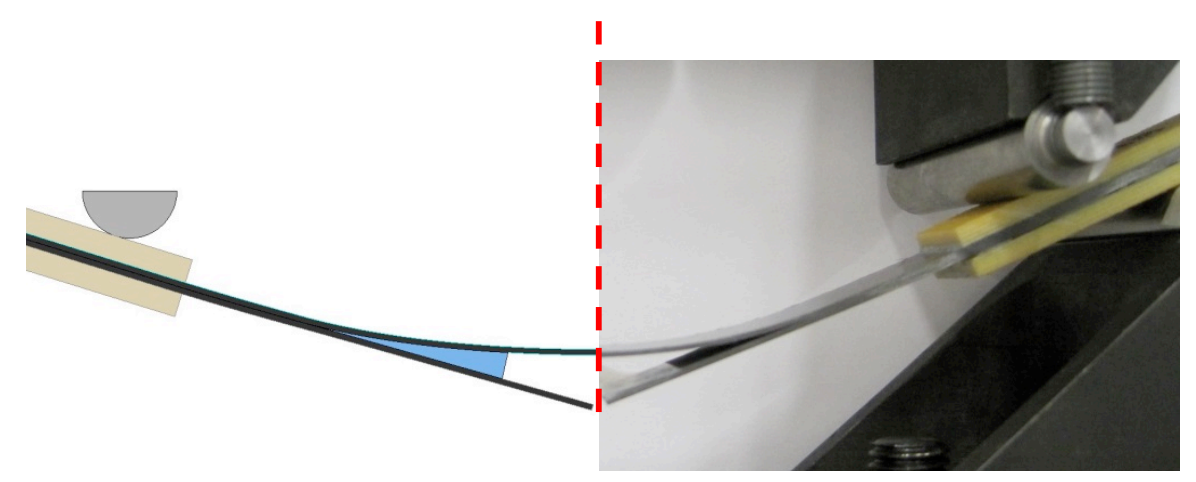

Figure 14: Detailed FE model including roller/tab contact. 


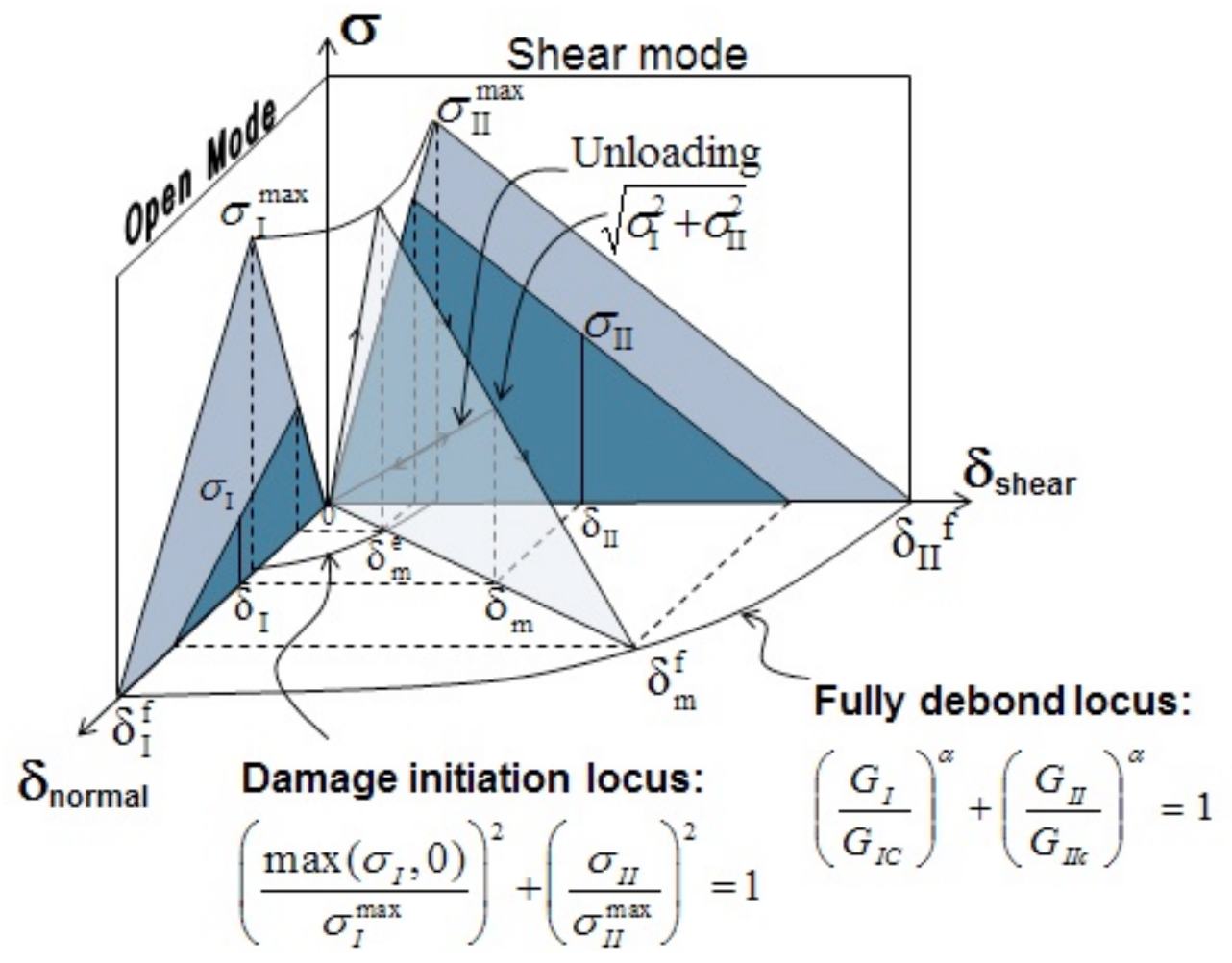

Figure 15: Mixed mode bi-linear traction-displacement curve [31].

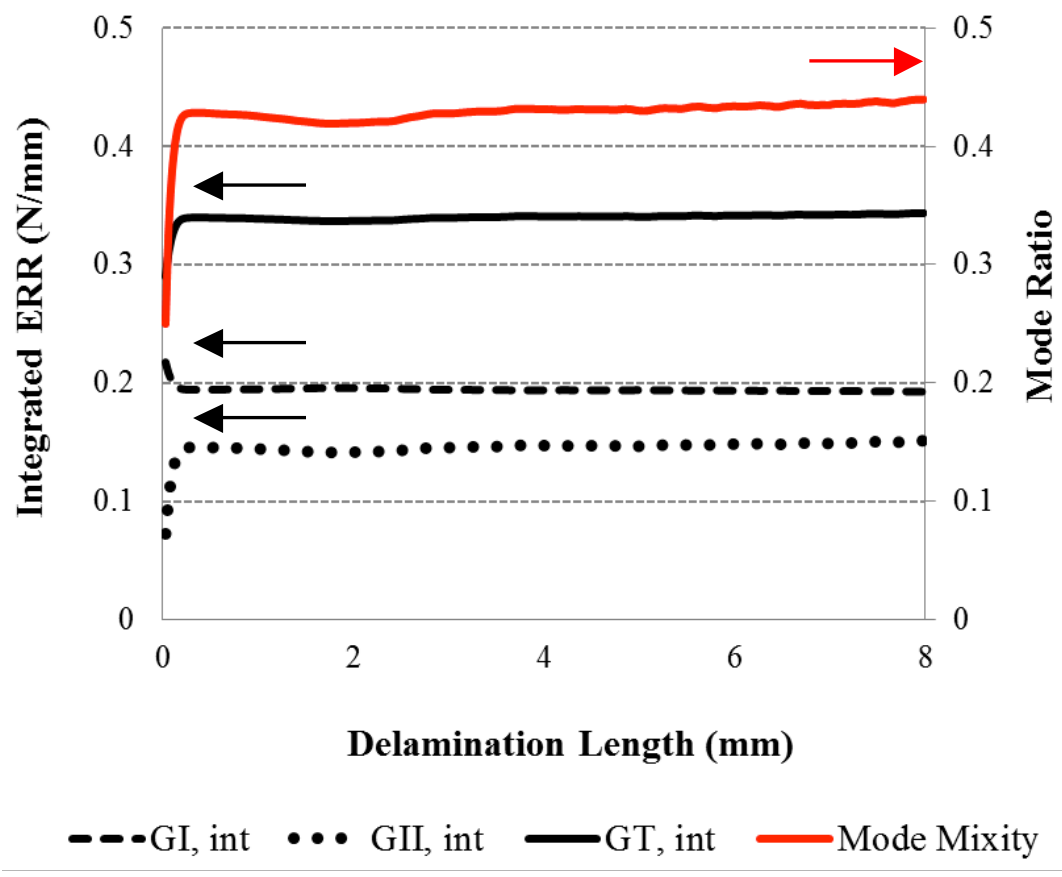

Figure 16: ERRs and mode-mixity values versus delamination length obtained from the finite element model. 
Table 1: Interlaminar fracture toughness $G_{C}$ for IM7/8552 at 0.43 mode mixity; number of specimens tested between brackets

\begin{tabular}{c|cc|cc}
\hline \hline \multicolumn{2}{c|}{ Initiation $G_{\mathbf{C}}\left(\mathbf{k J} / \mathbf{m}^{2}\right)$} & \multicolumn{2}{c}{ Propagation $\boldsymbol{G}_{\mathbf{C}}\left(\mathbf{k J} / \mathbf{m}^{2}\right)$} \\
\hline Rollers & NPC $^{(\mathrm{a})}$ & $\mathbf{P C}^{(\mathrm{b})}$ & $\mathbf{N P C}^{(\mathrm{a})}$ & PC $^{(\mathrm{b})}$ \\
\hline Smooth & $0.37 \pm 0.03(3)$ & $0.35 \pm 0.01(3)$ & $0.32 \pm 0.01(3)$ & $0.36 \pm 0.03(3)$ \\
\hline Grooved & $0.38 \pm 0.04(3)$ & $0.37 \pm 0.03(3)$ & $0.38 \pm 0.03(3)$ & $0.39 \pm 0.02(3)$ \\
\hline \hline
\end{tabular}

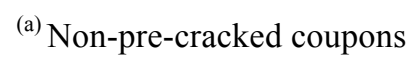

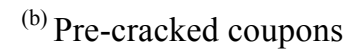

Table 2: Elastic properties of IM7/8552.

\begin{tabular}{ccccccccc}
\hline \hline $\begin{array}{c}\mathbf{E}_{11} \\
(\mathbf{M P a})\end{array}$ & $\begin{array}{c}\mathbf{E}_{22} \\
(\mathbf{M P a})\end{array}$ & $\begin{array}{c}\mathbf{E}_{33} \\
(\mathbf{M P a})\end{array}$ & $\boldsymbol{v}_{\mathbf{1 2}}$ & $\boldsymbol{v}_{\mathbf{1 3}}$ & $\boldsymbol{v}_{\mathbf{2 3}}$ & $\begin{array}{c}\mathbf{G}_{12} \\
(\mathbf{M P a})\end{array}$ & $\begin{array}{c}\mathbf{G}_{13} \\
(\mathbf{M P a})\end{array}$ & $\begin{array}{c}\mathbf{G}_{23} \\
(\mathbf{M P a})\end{array}$ \\
\hline 161000 & 11380 & 11380 & 0.32 & 0.32 & 0.436 & 5170 & 5170 & 3980 \\
\hline \hline
\end{tabular}


Table 3: Interlaminar cohesive properties of IM7/8552.

\begin{tabular}{ccccccc}
\hline $\begin{array}{c}G_{I C} \\
(\mathbf{N} / \mathbf{m m})\end{array}$ & $\begin{array}{c}G_{I I C}=G_{I I C} \\
(\mathbf{N} / \mathbf{m m})\end{array}$ & $\begin{array}{c}\sigma_{I}^{\max } \\
(\mathbf{M P a})\end{array}$ & $\begin{array}{c}\sigma_{I I}^{\max }=\sigma_{I I I}^{\max } \\
(\mathbf{M P a})\end{array}$ & $\begin{array}{c}K_{I} \\
\left(\mathbf{N} / \mathbf{m m}^{\mathbf{3}}\right)\end{array}$ & $\begin{array}{c}K_{I I}=K_{I I I} \\
\left(\mathbf{N} / \mathbf{m m}^{\mathbf{3}}\right)\end{array}$ & $n$ \\
\hline 0.24 & 0.78 & 111 & 120 & 467000 & 175000 & 0 \\
\hline \hline
\end{tabular}

Table 4: Comparison between the experimental results using analytical solutions and FEA.

\begin{tabular}{cccc}
\hline Method & $\begin{array}{c}\text { Bending Moment per } \\
\text { unit width }(\mathbf{N})\end{array}$ & $\begin{array}{c}\text { Mode Mixity } \\
(\boldsymbol{\phi})\end{array}$ & $\begin{array}{c}\text { Fracture } \\
\text { toughness }\left(\mathbf{k J} / \mathbf{m}^{\mathbf{2}}\right)\end{array}$ \\
\hline $\begin{array}{c}\text { Experimental data and } \\
\text { analytical solution }\end{array}$ & 50.86 & 0.43 & 0.38 \\
\hline FEA & 49 & 0.43 & 0.34 \\
\hline \hline
\end{tabular}

